\title{
Applications of artificial intelligence technologies in COVID-19 research:
}

\author{
A bibliometric study \\ Md Mahbub Hossain, ${ }^{1,2}$ Shah Akib Sarwar, ${ }^{3}$ E. Lisako J. McKyer, ${ }^{1}$ Ping Ma ${ }^{1}$
}

\begin{abstract}
The novel coronavirus disease (COVID-19) pandemic has impacted health and wellbeing globally. To strengthen preventive and clinical care amid this pandemic, technological innovations like artificial intelligence (AI) are increasingly used in different contexts. This bibliometric study aimed to assess the current scholarly development and prominent research domains in applications of AI technologies in COVID-19 research. A total of 105 articles were retrieved from MEDLINE database that emphasized on the use of AI in the context of COVID19. Most articles had multiple authors with a collaboration index of 7.18. Moreover, most of the articles were produced from the USA $(22.86 \%)$ and China $(21.9 \%)$, whereas developing countries were underrepresented among the contributing nations. Furthermore, several research domains were identified, including prevention and control, diagnostics, epidemiological characteristics, therapeutics, psychological conditions, and different areas of data sciences related to COVID-19. The current bibliometric evidence shows the early stage of development in this field, which necessitates equitable applications of AI in COVID-19 research emphasizing on health disparities, socio-legal issues, vaccine development, and applied public health research in this pandemic.
\end{abstract}

Keywords: COVID-19; Coronavirus; Artificial intelligence; Machine learning; Data mining

1. Department of Health Promotion and Community Health Sciences, School of Public Health, Texas A\&M University, College Station, TX 88743, USA.

2. Nature Study Society of Bangladesh, Khulna 09000, Bangladesh.

3. J. Mike Walker '66 Department of Mechanical Engineering, College of Engineering, Texas A\&M University, College Station, TX 88743, USA.

Corresponding author: Md Mahbub Hossain, Department of Health Promotion and Community Health Sciences, School of Public Health, Texas A\&M University, College Station, TX 88743, USA. Ph: +1-979-739-7014, Email: mhossain@tamu.edu 


\section{Introduction}

Since the novel coronavirus disease 2019 (COVID-19) outbreak started in December 2019, the global number of identified cases and mortality attributable to this pandemic have been increasing over time [1]. Later it was declared as a public health emergency and a global pandemic, highlighting the significance of the disease in the global context [1,2]. This pandemic has shown critical weaknesses of global health systems in terms of responding to public health emergencies [3]. To improve health systems preparedness for COVID-19 and future public health crises, it is essential to leverage technological advancements that may help in preventing outbreaks, detecting cases, tracing contacts, diagnosing clinical conditions, evaluating therapeutic and public health strategies, and predicting health outcomes in individuals and populations [4-6].

In recent years, artificial intelligence (AI) technologies are increasingly used to leverage computational approaches that stimulate or mimic cognitive processes, including reasoning, adaptation, sensory assessment, deep learning, and meaningful interactions [7,8]. Scholarly contributions from diverse fields of knowledge such as computer sciences, mathematics, biology, and psychological sciences have informed the development of AI, which is widely used in modeling, replicating, understanding, and predicting complex problems, processes, and outcomes in health sciences $[9,10]$. The use of AI in modern medicine is well documented in several metaanalyses in different clinical sub-specialties [11-13]. In the era of COVID-19, a growing interest in the use of AI developed in the global health community. A recent systematic review identified 51 studies representing 66 AI models that predicting hospital admission from pneumonia-related events, COVID-19 infection, length of hospital stay, progression to severe disease, and mortality risks in this pandemic [14]. As AI is emerging as a useful tool in this pandemic, it is necessary to assess the scientific development in this field by evaluating the growth of scholarly publications that inform how AI is being used in the context of COVID-19.

Scientometric assessments such as bibliometric studies are widely used to quantitatively evaluate the scientific publications in a given domain of research and inform the overall growth and pattern of development in that domain $[9,15]$. Such assessments are critical for prioritizing research and mobilizing resources for addressing research gaps in different branches of knowledge. Several bibliometric studies have shown the evolution of AI applications in different 
fields of health sciences $[9,16,17]$. Moreover, emerging bibliometric studies have reported how COVID-19 related research is increasingly produced globally [18-20]. However, to the best of our knowledge, no bibliometric study is available that reports the applications of AI in COVID19 related research. This study aimed to address this knowledge gap by a bibliometric evaluation of the current publications on the use of AI in COVID-19 research. Specifically, this study assessed the characteristics of relevant studies, top authors and sources of scholarly publications, and common research domains through keyword mapping on applications of AI technologies in COVID-19 related research publications.

\section{Methods}

In this study, we retrieved bibliometric data from the MEDLINE database using a set of keywords related to AI and COVID-19 (Table 1). After extracting the available literature, we reviewed the titles and abstracts of those articles to assess their eligibility for bibliometric analysis. Articles were excluded if they did not refer to any of the AI-related keywords or did not focus on COVID-19. Due to a limited number of publications, articles irrespective of their study design were included in this study with no language restrictions. As the COVID-19 outbreak started in December 2019, the search strategy was limited to 2019 and 2020.

\section{Table 1: Search strategy}

\begin{tabular}{ll}
\hline Search query & Title, abstract, subject heading (MeSH) \\
\hline Set 1: & "COVID-19" OR "2019-nCoV" OR "2019 coronavirus" OR "Wuhan \\
COVID-19 & coronavirus" OR "Wuhan virus" OR "Wuhan pneumonia" OR "2019 novel \\
related keywords & coronavirus" OR "novel coronavirus" OR "SARS-CoV-2" \\
\hline Set 2: & "Artificial intelligence" OR "Machine intelligence" OR "Artificial neutral \\
AI-related & network" OR "Recurrent neural network" OR "Machine learning" OR \\
keywords & "Generative adversarial network" OR "Deep learning" OR "Supervised \\
& learning" OR "Unsupervised learning" OR "Semi-supervised learning" OR \\
& "Reinforced learning" OR "Natural language processing" OR "Robotic" \\
& OR "Thinking computer system" OR "Fuzzy expert system" OR \\
& "Evolutionary computation" OR "Hybrid intelligent system" OR "Data \\
& mining" OR "Text mining" OR "Robotics" OR "Image recognition" OR \\
\hline Final & "Speech processing" \\
search query & 1 AND 2 \\
\hline
\end{tabular}


At the next stage, a descriptive summary of the key bibliometric characteristics was prepared, which included the frequency of the documents, sources, keywords, author appearance, coauthors per document, collaboration index, top ten authors, top countries affiliated with first author of respective documents, and journals that published AI-related articles on COVID-19.

Further, the co-occurrence of keywords was assessed to identify common research topics and connectivity within those topics using a mapping approach. Such keywords analysis is widely used in bibliometric studies to identify research hotspots within a given domain of knowledge, which informs what are the common topics represented in the literature and how those topics are related to each other $[9,20]$. A co-citation analysis was not performed considering the early stage of COVID-19 research and a low number of studies related to AI. In this study, reference management, statistical analyses, and keywords mapping were conducted using RefWorks, $R$ (Version 3.6.1), and VOSViewer software, respectively.

\section{Results}

A total of 105 articles were identified that met the criteria for this study. The summary of the bibliometric findings (Table 2) shows that these articles were published in 78 sources, and they had a total of 156 keywords. These documents were authored by 717 authors, whereas most of the articles had multiple authors $(n=711)$ with about seven authors per document and a collaboration index of 7.18 .

Table 1: Summary of the bibliometric findings

\begin{tabular}{|l|l|}
\hline Description & Results (frequency) \\
\hline Total published studies & 105 \\
\hline Sources (Journals, reports, books, etc.) & 78 \\
\hline Authors' keywords & 156 \\
\hline Total authors & 717 \\
\hline Documents with multiple authors & 711 \\
\hline Authors per document & 6.83 \\
\hline Co-authors per document & 7.69 \\
\hline Collaboration index & 7.18 \\
\hline
\end{tabular}


Further, the top ten authors, journals, and countries of primary affiliations were identified (Table 3). Among the top ten authors, Li L and Wang L had the highest number of publications ( $\mathrm{n}=5$ for each author) followed by Yang Y and Zhang L ( $n=4$ for each author). Moreover, the International Journal of Environmental Research and Public Health, JMIR Public Health and Surveillance, and Radiology journals published the highest ( $\mathrm{n}=4$ for each journal) number of articles on AI and COVID-19. Furthermore, among the countries with highest scientific production on AI application in COVID-19 research, the USA had highest number of articles ( $\mathrm{n}$ $=24)$ followed by China $(n=23)$, Italy $(n=8)$, Canada $(n=8)$, and India $(n=7)$.

Table 2: Top authors, journals, and originating countries

\begin{tabular}{|l|l|l|}
\hline \multicolumn{2}{|l|}{$\begin{array}{l}\text { Number of } \\
\text { documents }\end{array}$} & Percentage \\
\hline Top ten authors & 5 & $4.76 \%$ \\
\hline Li L. & 5 & $4.76 \%$ \\
\hline Wang L. & 4 & $3.81 \%$ \\
\hline Yang Y. & 4 & $3.81 \%$ \\
\hline Zhang L. & 3 & $2.86 \%$ \\
\hline Li H. & 3 & $2.86 \%$ \\
\hline Li S. & 3 & $2.86 \%$ \\
\hline Shi Y. & 3 & $2.86 \%$ \\
\hline Tian J. & 3 & $2.86 \%$ \\
\hline Wang G. & 3 & $2.86 \%$ \\
\hline Wang J. & \multicolumn{2}{|l|}{} \\
\hline Top ten journals & 4 & $3.81 \%$ \\
\hline $\begin{array}{l}\text { International Journal of Environmental } \\
\text { Research and Public Health }\end{array}$ & \multicolumn{2}{|l|}{} \\
\hline JMIR Public Health and Surveillance & 4 & $3.81 \%$ \\
\hline Radiology & 4 & $3.81 \%$ \\
\hline Diabetes and Metabolic Syndrome & 3 & $2.86 \%$ \\
\hline IEEE Transactions of Medical Imaging & 3 & $2.86 \%$ \\
\hline Journal of Medical Internet Research & 3 & $2.86 \%$ \\
\hline $\begin{array}{l}\text { Journal of the American Medical Informatics } \\
\text { Association }\end{array}$ & 3 & $2.86 \%$ \\
\hline $\begin{array}{l}\text { Catheterization and Cardiovascular } \\
\text { Interventions }\end{array}$ & 2 & $1.9 \%$ \\
\hline Data in Brief & 2 & $1.9 \%$ \\
\hline Head and Neck & 24 & $1.9 \%$ \\
\hline Top ten countries of origin & $22.86 \%$ \\
\hline USA & \multicolumn{2}{|l|}{} \\
\hline \multicolumn{2}{|l|}{} \\
\hline
\end{tabular}




\begin{tabular}{|l|l|l|}
\hline China & 23 & $21.9 \%$ \\
\hline Italy & 8 & $7.62 \%$ \\
\hline Canada & 8 & $7.62 \%$ \\
\hline India & 7 & $6.67 \%$ \\
\hline Germany & 4 & $3.81 \%$ \\
\hline Netherlands & 3 & $2.86 \%$ \\
\hline UK & 3 & $2.86 \%$ \\
\hline Republic of Korea & 2 & $1.9 \%$ \\
\hline Turkey & 2 & $1.9 \%$ \\
\hline
\end{tabular}

In the keyword analysis, several thematic areas of COVID-19 research were identified (Figure 1). While keywords related to coronavirus and AI appeared in the center of the word cloud, different sub-domains are found to be clustered around these topics. First, a cluster on the prevention and control of COVID-19 had several co-occurring keywords that included personal protective equipment and disease transmission-related keywords. These keywords represented several studies that reported the use of AI for the prevention and control of COVID-19 in clinical settings. For example, Zheng and colleagues proposed an improved susceptible-infected (ISI) model to estimate the variety of the infection that used the natural language processing (NLP) module and the long short-term memory (LSTM) network for predicting COVID-19 infection and prevention [21]. Second, a cluster of keywords related to diagnostics included tomography, $\mathrm{x}$-ray, and diagnostic imaging. For example, Wang and colleagues described a fully automatic deep learning system for COVID-19 diagnostics by analyzing routinely used radiological data [22]. 


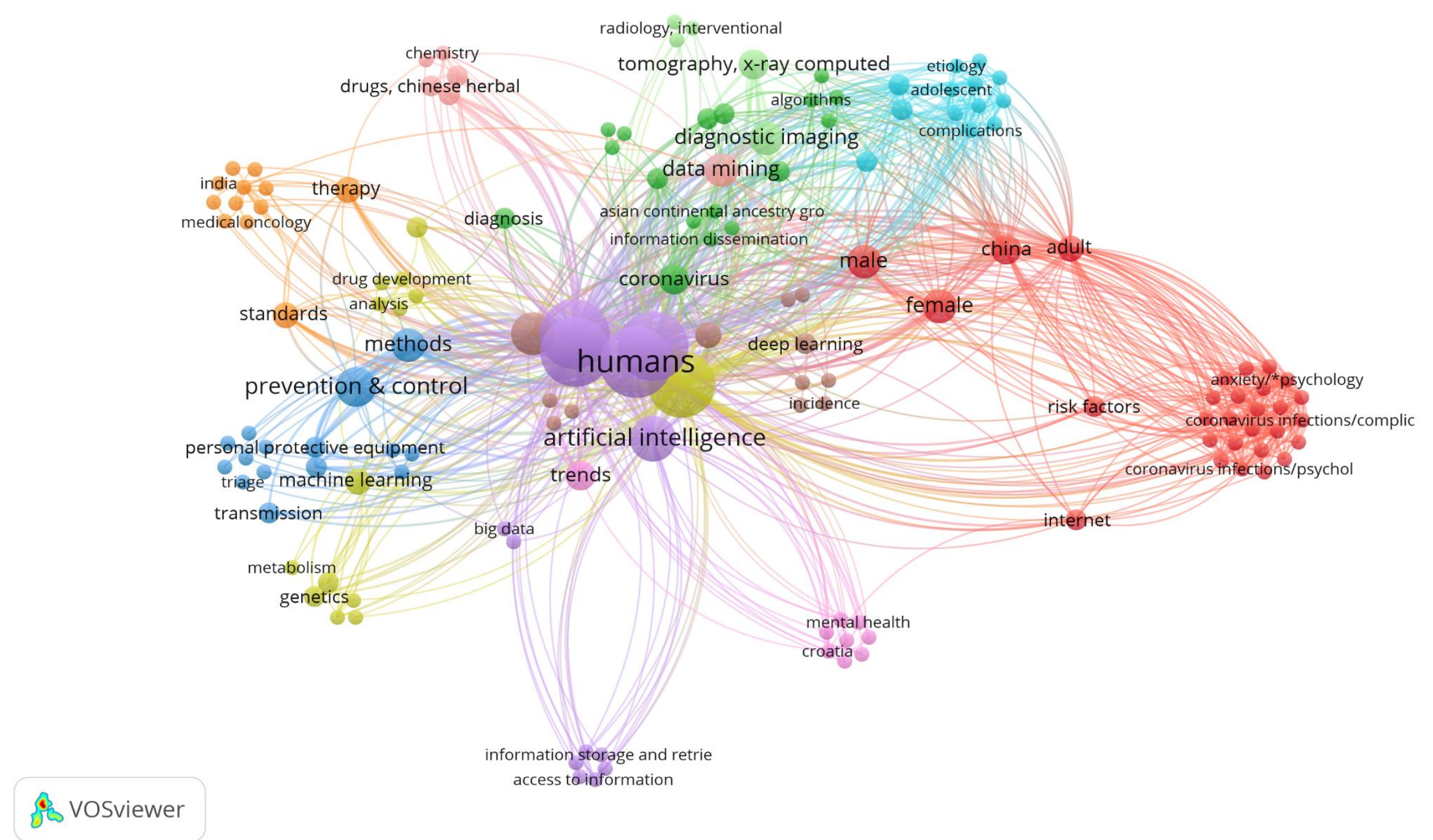

Figure 1: Keywords mapping of the applications of AI in COVID-19 related research 
Third, keywords related to the epidemiology of COVID-19 including etiological terms, age groups, ethnicity, and disease complications, created a cluster that highlighted the use of AI for etiological classifications among study samples. A study by Bai and colleagues assessed AI augmentation in radiological evaluation of the etiology of COVID-19 [23]. This study found a test accuracy of $96 \%$ (95\% confidence interval [CI]: 90-98\%), sensitivity of 95\% (95\% CI: 83$100 \%$ ) and specificity of $96 \%$ (95\% CI: 88-99\%) with Receiver Operating Characteristic (ROC) AUC of 0.95 and Precision-Recall (PR) AUC of 0.90. Fourth, therapy-related keywords formed two closely appearing clusters that included drug development, drug therapy, alternative drugs, and therapy for complications. For example, Gurwitz discussed the use of data mining for repurposing drugs for providing care to COVID-19 patients through analyzing electronic health records data [24]. Fifth, several keywords related to anxiety and other psychological issues developed a cluster highlighting a growing use of $\mathrm{AI}$ in coronavirus-related psychological research. For example, Li and colleagues analyzed social media (Weibo) posts from 17,865 users using the approach of Online Ecological Recognition (OER) based on several machine-learning predictive models for studying psychological impacts of COVID-19 [25]. Lastly, smaller clusters of less-commonly appearing keywords highlighted research domains that included evidencebased medicine, access to information, disasters, genetics, metabolism, and different areas of data sciences [26-28].

\section{Discussion}

This study assessed the bibliometric characteristics of the current scholarly literature on the application of AI in COVID-19 research. Despite a considerable expansion of COVID-19 publications, the number of articles on $\mathrm{AI}$ in this domain appears to be low, necessitating further research leveraging AI technologies in this pandemic. Moreover, a major proportion of the available literature originated from the US and China, whereas studies from South America, Australia, and Africa were considerably low. This can be attributable to the variations in the number of cases, research priorities, and institutional capacities of respective countries [20,29]. This informs a need for strengthening AI-related research in under-representing countries that are currently having a high burden of COVID-19 and have inadequate resources for research. Furthermore, the most prolific authors and journals provide insights on how the scientific 
community has been leading the use of AI in COVID-19 research, which may inform future researchers and journals to explore opportunities to make meaningful contributions through scholarly communications in this field. Another important finding of this study is the research domains consisting of frequently co-occurring keywords, which highlights how AI is being used in preventive, diagnostic, psychological, and epidemiological research in COVID-19. However, the keywords mapping also inform research gaps such as a lack of keywords on vaccine-related research, social aspects of COVID-19, and health systems [20]. These under-reported research domains should be explored in future applications of AI in COVID-19 research.

There are several bibliometric studies on COVID-19 publications [18-20]. To the best of our knowledge, this is the first one to evaluate the contemporary COVID-19 literature than emphasized on the applications of AI technologies. In recent years, scholars and practitioners are increasingly using AI in health sciences research that is facilitating the use of advanced analytics to better understand different aspects of human health and wellbeing [11,13]. However, the number of articles and limited research domains within the same suggests that the application of AI in COVID-19 research is a developing area in the global research landscape. This study provides bibliometric evidence on the early-stage of research in this field and calls for further research using AI exploring diverse research domains that may help policymakers and practitioners to make informed decisions during this pandemic. Also, ethical concerns, as well as safety and security of using patient data, are critical concerns in data sciences [30,31].

Prospective research using AI should acknowledge such issues addressing socio-legal issues that may arise in using COVID-19 data.

Another perspective on using AI in health sciences is promoting equity since the beginning of the AI-related research. Contemporary scholarly discourses highlight the fact that the use of AI does not consider potential pitfalls that may underestimate health disparities in marginalized populations or underrepresented groups [32,33]. Moreover, specific population groups may have unique health risks amid this pandemic, which includes older adults and people with co-morbid conditions [34-37]. In this regard, the use of AI should be leveraged to proactively identify complex health needs that may arise during this pandemic and address the same. As AI-related research in COVID-19 is still under development, we call for intellectual and professional conversations on equitable use of AI that prevents suboptimal use of such technologies and helps 
in addressing health disparities. Furthermore, current scholarly contributions of AI do not provide much insights on how such technologies are used for public health surveillance, mass communication, or applied public health practice. It is necessary to facilitate such applied research on AI to strengthen measures addressing public health challenges during this pandemic. The findings of this study also inform a critical lack of diversity in research domains and contexts, highlighting the need for global collaborations for multidisciplinary research. Such collaborations may bring the technical competencies of multiple knowledge domains from different contexts complementing knowledge exploration and synthesis in COVID-19 [20,30].

There are a few limitations of this bibliometric study that should be acknowledged. One such limitation is the selection of the MEDLINE database for this study. Although it is the most extensive database in the health sciences, it may not cover a complete collection of COVID-19 research. Therefore, the inclusion of other databases, especially a growing body of preprints available in the Google Scholar database, could have provided additional insights that are not available in our study. Another limitation of this study is a lack of citations and collaborations networks, which could not be developed due to a low number of studies and continuously changing citations metrics. Future bibliometric studies should address these limitations and examine how the use of AI in COVID-19 evolves over time.

\section{Conclusions}

COVID-19 pandemic has become a global health concern affecting health and wellbeing across populations. For effective decision-making, the scholars, practitioners, and policymakers need evidence on the etiology, clinical features, diagnosis, prognosis, health outcomes, and potential impacts of this pandemic on societies and systems. AI offers a wide range of opportunities to use the emerging data on COVID-19 and yield meaningful insights that may help the key decisionmakers amid this pandemic. Given the current state of scholarly development, the application of AI in COVID-19 research is still low and focused on fewer research domains, which necessitates further research on how different AI technologies can be used to leverage computational advantages in addressing uncertainties regarding this pandemic. It is essential to prioritize applications of AI to facilitate research-driven decision-making and ensure global advancements in this field are optimally used for promoting health and saving lives in the era of COVID-19. 
Funding: None.

Author contributions: MMH and SAS conceptualized this study. MMS and ELJM designed the methodology. MMH and SAS retrieved and reviewed the literature. MMH and PM analyzed the data. MMH prepared the first draft of the manuscript. SAS, ELJM, and PM reviewed and edited the first draft. All authors reviewed and approved the submitted version of the manuscript.

Conflicts of interest: The authors declare no conflicts of interest. 


\section{Reference}

[1] World Health Organization. Coronavirus Disease (COVID-19) Situation Reports 2020. https://www.who.int/emergencies/diseases/novel-coronavirus-2019/situation-reports/ (accessed June 11, 2020).

[2] World Health Organization. Coronavirus (COVID-19) events as they happen 2020. https://www.who.int/emergencies/diseases/novel-coronavirus-2019/events-as-they-happen (accessed June 11, 2020).

[3] Shamasunder S, Holmes SM, Goronga T, Carrasco H, Katz E, Frankfurter R, et al. COVID-19 reveals weak health systems by design: Why we must re-make global health in this historic moment. Glob Public Health 2020:1-7.

https://doi.org/10.1080/17441692.2020.1760915.

[4] Abeler J, Bäcker M, Buermeyer U, Zillessen H. Covid-19 contact tracing and data protection can go together. JMIR MHealth UHealth 2020;8. https://doi.org/10.2196/19359.

[5] Moore JH, Barnett I, Boland MR, Chen Y, Demiris G, Gonzalez-Hernandez G, et al. Ideas for how informaticians can get involved with COVID-19 research. BioData Min 2020;13:3. https://doi.org/10.1186/s13040-020-00213-y.

[6] Mahmood S, Hasan K, Colder Carras M, Labrique A. Global Preparedness Against COVID-19: We Must Leverage the Power of Digital Health. JMIR Public Heal Surveill 2020;6:e18980. https://doi.org/10.2196/18980.

[7] Jiang F, Jiang Y, Zhi H, Dong Y, Li H, Ma S, et al. Artificial intelligence in healthcare: Past, present and future. Stroke Vasc Neurol 2017;2:230-43. https://doi.org/10.1136/svn2017-000101.

[8] Fellous JM, Sapiro G, Rossi A, Mayberg H, Ferrante M. Explainable Artificial Intelligence for Neuroscience: Behavioral Neurostimulation. Front Neurosci 2019;13. https://doi.org/10.3389/fnins.2019.01346.

[9] Tran B, Vu G, Ha G, Vuong Q-H, Ho M-T, Vuong T-T, et al. Global Evolution of Research in Artificial Intelligence in Health and Medicine: A Bibliometric Study. J Clin Med 2019;8:360. https://doi.org/10.3390/jcm8030360.

[10] Deo RC. Machine learning in medicine. Circulation 2015;132:1920-30. https://doi.org/10.1161/CIRCULATIONAHA.115.001593.

[11] Islam MM, Poly TN, Walther BA, Yang HC, Li Y-C (Jack). Artificial Intelligence in Ophthalmology: A Meta-Analysis of Deep Learning Models for Retinal Vessels Segmentation. J Clin Med 2020;9:1018. https://doi.org/10.3390/jcm9041018.

[12] Moon SJ, Hwang J, Kana R, Torous J, Kim JW. Accuracy of machine learning algorithms for the diagnosis of autism spectrum disorder: Systematic review and meta-analysis of brain magnetic resonance imaging studies. J Med Internet Res 2019;21. https://doi.org/10.2196/14108.

[13] Lao Y, Jia B, Yan P, Pan M, Hui X, Li J, et al. Diagnostic accuracy of machine-learning- 
assisted detection for anterior cruciate ligament injury based on magnetic resonance imaging: Protocol for a systematic review and meta-analysis. Med (United States) 2019;98. https://doi.org/10.1097/MD.0000000000018324.

[14] Wynants L, Van Calster B, Bonten MMJ, Collins GS, Debray TPA, De Vos M, et al. Prediction models for diagnosis and prognosis of covid-19 infection: Systematic review and critical appraisal. BMJ 2020;369. https://doi.org/10.1136/bmj.m1328.

[15] Hossain MM. Umbrella Review as an Emerging Approach of Evidence Synthesis in Health Sciences: A Bibliometric Analysis. SSRN Electron J 2020. https://doi.org/10.2139/ssrn.3551055.

[16] Tran BX, McIntyre RS, Latkin CA, Phan HT, Vu GT, Nguyen HLT, et al. The current research landscape on the artificial intelligence application in the management of depressive disorders: A bibliometric analysis. Int J Environ Res Public Health 2019;16. https://doi.org/10.3390/ijerph16122150.

[17] Chen X, Chen J, Cheng G, Gong T. Topics and trends in artificial intelligence assisted human brain research. PLoS One 2020;15. https://doi.org/10.1371/journal.pone.0231192.

[18] Chahrour M, Assi S, Bejjani M, Nasrallah AA, Salhab H, Fares MY, et al. A Bibliometric Analysis of COVID-19 Research Activity: A Call for Increased Output. Cureus 2020;12. https://doi.org/10.7759/cureus.7357.

[19] Kambhampati SBS, Vaishya R, Vaish A. Unprecedented surge in publications related to COVID-19 in the first three months of pandemic: A bibliometric analytic report. J Clin Orthop Trauma 2020;11:S304. https://doi.org/10.1016/j.jcot.2020.04.030.

[20] Hossain MM. Current status of global research on novel coronavirus disease (COVID-19): a bibliometric analysis and knowledge mapping. F1000Research 2020;9:374. https://doi.org/10.12688/f1000research.23690.1.

[21] Zheng N, Du S, Wang J, Zhang H, Cui W, Kang Z, et al. Predicting COVID-19 in China Using Hybrid AI Model. IEEE Trans Cybern 2020. https://doi.org/10.1109/TCYB.2020.2990162.

[22] Wang S, Zha Y, Li W, Wu Q, Li X, Niu M, et al. A Fully Automatic Deep Learning System for COVID-19 Diagnostic and Prognostic Analysis. Eur Respir J 2020. https://doi.org/10.1183/13993003.00775-2020.

[23] Bai HX, Wang R, Xiong Z, Hsieh B, Chang K, Halsey K, et al. AI Augmentation of Radiologist Performance in Distinguishing COVID-19 from Pneumonia of Other Etiology on Chest CT. Radiology 2020:201491. https://doi.org/10.1148/radiol.2020201491.

[24] Gurwitz D. Repurposing current therapeutics for treating COVID-19: A vital role of prescription records data mining. Drug Dev Res 2020. https://doi.org/10.1002/ddr.21689.

[25] Li S, Wang Y, Xue J, Zhao N, Zhu T. The Impact of COVID-19 Epidemic Declaration on Psychological Consequences: A Study on Active Weibo Users. Int J Environ Res Public Health 2020;17. https://doi.org/10.3390/ijerph17062032. 
[26] Rada G, Verdugo-Paiva F, Ávila C, Morel-Marambio M, Bravo-Jeria R, Pesce F, et al. Evidence synthesis relevant to COVID-19: a protocol for multiple systematic reviews and overviews of systematic reviews. Medwave 2020;20:e7868.

https://doi.org/10.5867/medwave.2020.03.7867.

[27] Randhawa GS, Soltysiak MPM, El Roz H, de Souza CPE, Hill KA, Kari L. Machine learning using intrinsic genomic signatures for rapid classification of novel pathogens: COVID-19 case study. PLoS One 2020;15:e0232391. https://doi.org/10.1371/journal.pone.0232391.

[28] Tárnok A. Machine Learning, COVID-19 (2019-nCoV), and multi-OMICS. Cytometry A 2020;97:215-6. https://doi.org/10.1002/cyto.a.23990.

[29] Hossain MM, Sultana A, Tasnim S, Fan Q, Ma P, McKyer ELJ, et al. Prevalence of mental disorders among people who are homeless: An umbrella review. Int J Soc Psychiatry 2020:002076402092468. https://doi.org/10.1177/0020764020924689.

[30] Hossain MM, Sharma R, Sultana A, Tasnim S, Faizah F. Globalising artificial intelligence for improved clinical practice. Indian J Med Ethics 2019:01-2.

https://doi.org/10.20529/ijme.2019.074.

[31] Hossain MM, Hong YA. Trends and characteristics of protected health information breaches in the United States. AMIA. Annu Symp Proceedings AMIA Symp 2019;2019:1081-90.

[32] Gianfrancesco MA, Tamang S, Yazdany J, Schmajuk G. Potential Biases in Machine Learning Algorithms Using Electronic Health Record Data. JAMA Intern Med 2018;178:1544-7. https://doi.org/10.1001/jamainternmed.2018.3763.

[33] Parikh RB, Teeple S, Navathe AS. Addressing Bias in Artificial Intelligence in Health Care. JAMA - J Am Med Assoc 2019;322:2377-8. https://doi.org/10.1001/jama.2019.18058.

[34] Hossain MM, Mazumder H, Tasnim S, Nuzhath T, Sultana A. Geriatric Health in Bangladesh during COVID-19: Challenges and Recommendations. J Gerontol Soc Work 2020:1-4. https://doi.org/10.1080/01634372.2020.1772932.

[35] Mazumder H, Hossain MM, Das A. Geriatric Care during Public Health Emergencies: Lessons Learned from Novel Corona Virus Disease (COVID-19) Pandemic. J Gerontol Soc Work 2020. https://doi.org/10.1080/01634372.2020.1746723.

[36] Raifman MA, Raifman JR. Disparities in the Population at Risk of Severe Illness From COVID-19 by Race/Ethnicity and Income. Am J Prev Med 2020. https://doi.org/10.1016/j.amepre.2020.04.003.

[37] Hossain MM, Tasnim S, Sultana A, Faizah F, Mazumder H, Zou L, et al. Epidemiology of mental health problems in COVID-19: a review. PsyArXiv 2020. https://doi.org/10.31234/osf.io/q8e5u. 